\title{
A Case of Diabetic Amyotrophy Associated with 3243 Mitochondrial tRNA(leu; UUR) Mutation and Successful Therapy with Coenzyme Q10
}

\author{
YoSHIHIKO SUZUKI, HIROKO KADOWAKI*, YOSHIHITO ATSUMI, \\ KAZUHIRO HOSOKAWA, HIDEKI KATAGIRI**, TAKASHI KADOWAKI**, \\ YOSHITOMO OKA**, KeIKO UYAMA, ATSUKO MOKUBO, \\ TAKAYUKI ASAHINA, CHISATO MURATA AND KEMPEI MATSUOKA \\ Saiseikai Central Hospital, Tokyo 108, *Institute for Diabetes Care and Research, \\ Asahi Life Foundation, Tokyo 160, and **Third Department of Internal Medicine, \\ Faculty of Medicine, University of Tokyo, Tokyo 113, Japan
}

\begin{abstract}
We report the case of 71-year-old male who was once diagnosed as having diabetic amyotrophy, because of pronounced wasting in proximal muscles, massive weight loss, and development of paresthesia in his legs. Afterwards, ragged red fibers and mitochondrial tRNA mutation at position 3243 were documented in muscle biopsy. He had diabetes mellitus associated with 3243 mitochondrial DNA mutation, suggesting that clinically, diabetic amyotrophy may be overlapped with mitochondria-related disease entities in some parts. Coenzyme Q10 administration was effective in relieving the symptoms in his legs, fatigue, and residual urine in his bladder. These were confirmed with the improvement in neurological parameters.

In conclusion, this case gives important help in understanding myopathy in diabetes. It would be important to check on the 3243 mitochondrial tRNA mutation in patients with diabetic amyotrophy and/or diabetic neuropathic symptoms.
\end{abstract}

Key words: Diabetic amyotrophy, Mitochondrial myopathy, Coenzyme Q10, Diabetic peripheral neuropathy, 3243 mitochondrial tRNA mutation

(Endocrine Journal 42: 141-145, 1995)

MITOCHONDRIAL disorder is known to be characterized by various neuro-muscular dysfunctions, as in MELAS (mitochondrial myopathy, encephalopathy, lactic acidosis, and stroke-like episodes), MERRF (myoclonic epilepsy with ragged red fibers) and KSS (Kearns-Sayre syndrome) [1]. Recently, a new subtype was reported by Ouweland et al., which showed diabetes and/or hearing loss, without the hallmarks of the above wellknown syndromes [2]. The phenotype was associated with an $A \rightarrow G$ mutation of the mitochondrial tRNA (leucine; UUR) at position 3243 [3-5].

Received: April 25, 1994

Accepted: October 26, 1994

Correspondence to : Dr. Yoshihiko SUZUKI, Saiseikai Central Hospital, 1-4-17 Mita, Minato-ku, Tokyo 180, Japan
We recently treated a patient who was once diagnosed as having diabetic amyotrophy (DA), but thereafter found to have the 3243 mitochondrial DNA (mtDNA) mutation in muscle. We herein describe the clinical and pathological findings and successful therapy.

\section{Case}

The case was a 71-year-old man. There was no consanguinity in his family. His mother had noninsulin dependent diabetes and died with renal failure. Neuromuscular diseases were unknown in her relatives. His father and his three children had no diabetes. 
At age 50, he had glycosuria and polydipsia, and was diagnosed as having diabetes mellitus by oral glucose tolerance test, with a fasting plasma glucose level of $115 \mathrm{mg} / \mathrm{d} l$. He developed diffuse paresthesia in his legs and there was muscle wasting around the thighs. Although diabetic control was achieved with diet only, his symptoms worsened.

In the next 3 years, his weight dropped from 52 $\mathrm{kg}$ to $45 \mathrm{~kg}$. Muscle weakness and wasting were more pronounced in the thigh than in the calf, with absence of the deep tendon reflex in the lower extremities.

From 64 years of age, he noticed mild hearing loss, diagnosed as presbycusis. The paresthesia and pain in his legs were aggravated, and we prescribed several neurergic medicines for him, but to no effect.

Since around 70 years of age, he noticed increasing difficulty in climbing stairs and looked disabled. His weight was $39 \mathrm{~kg}$. He was diagnosed as having diabetic amyotrophy, because he fulfilled the classical criteria of Garland [8]. Because the fasting plasma glucose was aggravated slightly, he received an oral hypoglycemic agent Gliclazide, $40 \mathrm{mg}$ daily, to obtain better glycemic control.

At 71 years of age, daily oral Coenzyme Q10 (CoQ) $150 \mathrm{mg}$ was prescribed to him. Four weeks

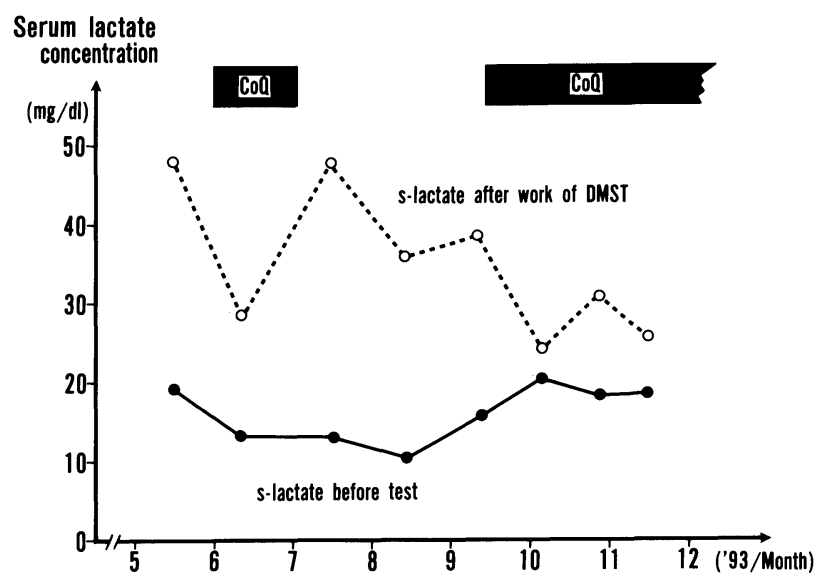

Fig. 1. Changes in serum lactate during the first CoQ therapy, after stopping CoQ therapy, and when restarting CoQ therapy. The solid line indicates the resting levels and the dashed line indicates the levels after double master stepping test. The serum lactate tended to decrease, particularly during the period of CoQ therapy. later, he noticed that the fatigue and leg paresthesia had disappeared, and there was improvement in serum lactacidemia (Fig. 1). To confirm the effect of the CoQ, CoQ therapy was stopped for 6 weeks and he again felt fatigue, and the serum lactate level after work rose to the previous level $(35.9-47.7 \mathrm{mg} / \mathrm{d} l)$. During the period of these observations, no other medication except daily Gliclazide, $40 \mathrm{mg}$, was prescribed. Fasting plasma glucose was around $140 \mathrm{mg} / \mathrm{dl}$, and $\mathrm{HbAlc}$ was around $8.0 \%$. There seemed to be no difference between these glycemic parameters before and after CoQ therapy.

In August, 1993, he was admitted to undergo muscle biopsy. On physical examination, there was diffuse wasting ( $162 \mathrm{~cm}, 39 \mathrm{~kg}$ ). His blood pressure was $130 / 74 \mathrm{mmHg}$, and heart rate was 84 beats $/ \mathrm{min}$. Consciousness and intelligence seemed normal for his age. No ptosis or external ophthalmoplegia was observed. Visual acuity was normal. Bilateral hearing loss was so severe that he needed hearing aid in daily life. Gripping power was weakened (right hand $24 \mathrm{~kg}$, left hand $24 \mathrm{~kg}$ ). Chest and lumbosacral spine roentgenograms were within normal limits. Motor nerve conduction velocities in tibial and median nerves were delayed, being $40.3 \mathrm{~m} / \mathrm{sec}$ and $58.1 \mathrm{~m} / \mathrm{sec}$ respectively (Fig. 2). No retinal bleeding was observed. His electrocardiogram was normal. Urinary retention after

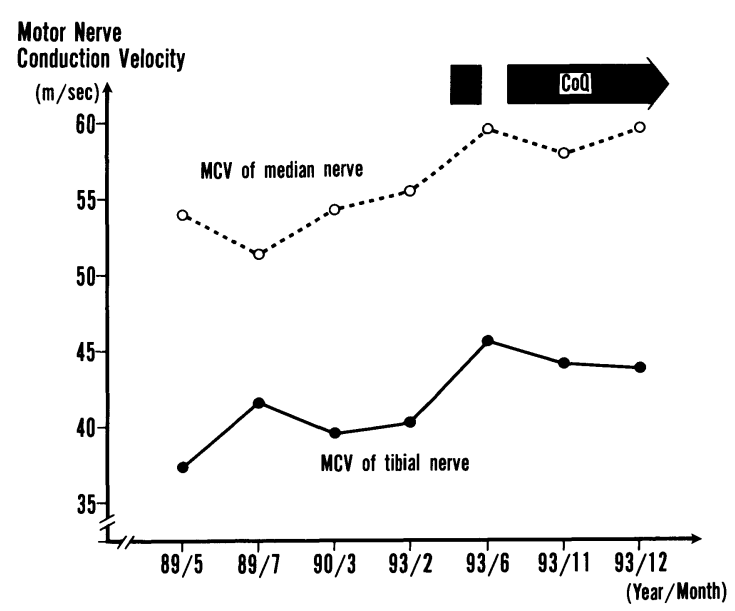

Fig. 2. Difference between motor nerve conduction velocities in tibial and median nerves before and after CoQ therapy. The velocities improved after starting $\mathrm{CoQ}$ therapy. 
voiding was seen on cystopyelography (Fig. 3).

Fasting plasma glucose was $130 \mathrm{mg} / \mathrm{dl}, \mathrm{HbA} 1 \mathrm{c}$ being $8.3 \%, 24$ h-urinary C-peptide reactivity being $47 \mu \mathrm{g}$. Total and differential white blood cell counts, hemoglobin, blood urea nitrogen, serum electrolytes, creatine kinase, creatinine and cholesterol were within normal limits. No $3243 \mathrm{mtDNA}$ mutation was not found in blood leukocytes even on frequent examination [4].

On muscle biopsy, he gave informed consent.

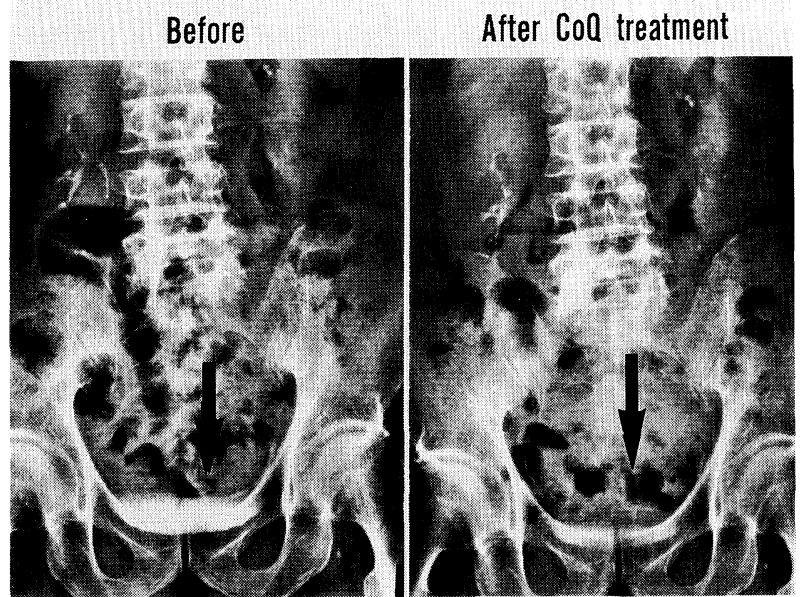

Fig. 3. Difference between residual urine on cystopyelography before and after CoQ therapy. The residual volume decreased after starting CoQ therapy.
On light microscopic examination, a biopsy specimen for the biceps brachii muscle showed mild myogenic change and ragged red fibers (RRF) on modified Gomori-trichrome staining (Fig. 4) [6]. On $\mathrm{NADH}$-Tetrazolium reductase stain, intermyofibriller networks were disorganized in $20 \%$ of the type 1 fibers; there were many 'moth-eaten' fibers and whorled fibers. On succinic dehydrogenase (SDH) stain, $5 \%$ of RRFs were stained strongly and a few strongly $\mathrm{SDH}$-reactive blood vessels were noted. On cytochrome c oxidase (CCO) stain, 7$8 \%$ of the fibers had no enzyme activity, showing focal CCO deficiency. On ATPase staining, type 1, $2 \mathrm{~A}, 2 \mathrm{~B}$ and $2 \mathrm{C}$ were $43 \%, 50 \%, 6 \%$ and $1 \%$, respectively. Type $2 \mathrm{~B}$ fibers were decreased in number and there was type $2 \mathrm{~B}$ fiber atrophy.

Respiratory enzyme assay revealed that complex I activity (NADH cytochrome c reductase) was remarkably decreased to $5.0 \mathrm{nmol} / \mathrm{min} / \mathrm{mg}$ mitochondrial protein (normal $54.5 \pm 23.2, \mathrm{n}=5$ ), succinate cytochrome $\mathrm{c}$ reductase was within the normal limit of $89.5 \mathrm{nmol} / \mathrm{min} / \mathrm{mg}$-mitochondrial protein (normal $75.2 \pm 25.0, \mathrm{n}=5$ ), cytochrome $\mathrm{c}$ oxidase was slightly decreased to $21.0 \mathrm{nmol} / \mathrm{min} /$ mg-mitochondrial protein (normal $38.2 \pm 2.7, \mathrm{n}=5$ ).

Despite the result of mtDNA analysis of leukocytes, 3243 mtDNA mutation was documented, apparently in muscle. The method of analysis is

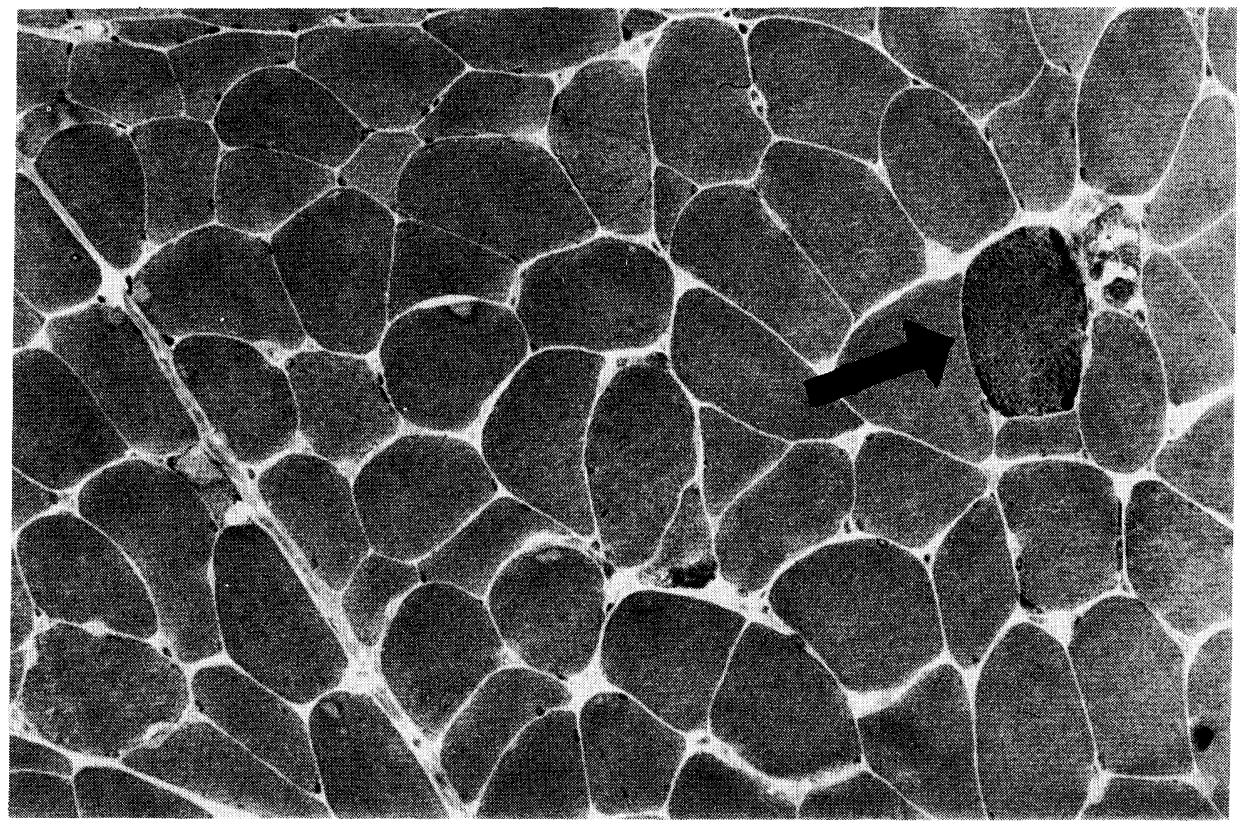

Fig. 4. Muscle pathology. There was a moderate variation in fiber size and ragged red fibers, showing the myopathic change on modified Gomori trichrome stain. (cf. Within this muscle, 3243 mitochondrial tRNA mutation was documented.) 
described elsewhere [6, 7].

After discharge, he re-started CoQ $150 \mathrm{mg}$ daily. One month later, he again felt relief from paresthesia in his legs. Weakness and fatigue disappeared. Three months later, the leg paresthesia and the urinary retention disappeared, but hearing loss remained.

Several examination data supported the improvement in his symptoms. The serum lactate level after work decreased, and the nerve conduction velocity in both tibial and median nerves improved (Figs. 1, 2). The urinary retention after voiding disappeared on cystopyelography (Fig. 3).

\section{Discussion}

For 100 years, diabetes has been incriminated as a cause of wasting and weakness of the pelvifemoral muscles [8-10, 11]. Garland termed this ailment as diabetic amyotrophy and defined it as "a syndrome which includes weakness and wasting of muscles with tendon areflexia, all associated with frank diabetes or at least with impaired glucose tolerance. Pain of a similar distribution to the amyotrophy is a frequent though not constant feature" [8].

It was therefore surprising that this patient, who was once judged to fulfill the criteria for DA, had the myopathic changes, RRF, strongly SDH-reactive vessels, decreased complex I activity and 3243 mtDNA mutation in muscle. Clinically we therefore assume that, in some cases, diabetic amyotrophy overlaps mitochondria-related disease entities [11, 12]. This may be supported partly by Nielsen et al. who reported that the changes in mitochondria are pronounced in some muscle biopsies from juvenile diabetics [13]. Furthermore, the fact that the onset in patients with DA generally occurs after the age of 50 , suggests a relationship between the worsening mitochondrial function and the age-related decline process [8].

Of clinical importance in this case is the discrepancy in finding the $3243 \mathrm{mtDNA}$ mutation between blood and muscle. This phenomenon indicated that a variable proportion of mutant mtDNA exists in various tissues even in a single individual, and is sometimes abundant in muscle and scarce in leukocytes. A diagnosis of the $3243 \mathrm{mtDNA}$ mutation therefore cannot be excluded even when this mutation is not identified in the DNA speci- men from the peripheral blood.

Neuropathy has been recognized as a clinical feature in several mitochondrial disorders, but in cases of diabetes mellitus associated with $3243 \mathrm{mtDNA}$ mutation, it has not been documented. The successful treatment of this patient with CoQ is therefore is important, suggesting that some neuropathy in diabetes overlaps with mitochondrial neuropathy and can respond to CoQ therapy.

Particularly in this case, CoQ administration was effective not only in relieving the symptoms of neuropathy, but also in reducing the lactate level and in relieving the fatigue and weakness, which were confirmed with the improvement in several parameters. The mechanism of CoQ is thought to be as follows: (1) CoQ supplementation might compensates for CoQ deficiency: (2) CoQ as an antioxidant might prevent membrane damage due to lipid peroxidation by free radicals, and (3) a high membrane concentration of $\mathrm{CoQ}$ increases membrane fluidity, favoring interaction between the components of the respiratory chain complexes. We could not determine, however, which of these mechanisms contributed most to his improvement, since we did not compare the pathophysiological basis of direct CoQ function before and after the treatment. The role of CoQ administration requires further elucidation [14].

In this patient, despite the remarkable improvement of various symptoms, neither hearing loss nor muscle wasting was relieved. This is probably due to irreversible alteration of the tissues affected.

In conclusion, this case gives important help in understanding the pathophysiology of myopathy and neuropathy in diabetes. It would be important to check on the 3243 mtDNA mutation in cases of diabetic amyotrophy and/or diabetic neuropathy. Further studies are needed to find clues to candidate responders to CoQ treatment beforehand.

\section{Acknowledgments}

The authors express their cordial thanks to Drs. Nobuyuki Murakami, Yu-ichi Goto and Ikuya Nonaka (Division of Ultrastructural Research, National Institute of Neuroscience, NCNP, Tokyo, Japan) for the precise examination of the muscle pathology, biochemistry, and analysis of 3243 mtDNA mutation. 


\section{References}

1. Gerbitz KD (1992) Does the mitochondrial DNA play a role in the pathogenesis of diabetes? Diabetologia 35: 1181-1186.

2. Van den Ouweland JMW, Lemkes HHPJ, Ruitenbeek W, Sandkuijl LA, de Vijlder ME, Struyvenberg PAA, van de Kamp JJP, Maassen JA (1992) Mutation in mitochondrial tRNA Leu (UUR) gene in a large pedigree with maternally transmitted type II diabetes mellitus and deafness. Nature genetics 1: 368-371.

3. Kadowaki T, Kadowaki H, Mori Y, Tobe K, Sakuta R, Suzuki Y, Tanabe Y, Sakura H, Awata T, Goto Y, Hayakawa T, Matsuoka K, Kawamori R, Kamada T, Horai S, Nonaka I, Hagura R, Akanuma Y, Yazaki $Y$ (1994) A subtype of diabetes mellitus associated with a mutation of mitochondrial DNA. N Eng J Med 330: 962-968.

4. Kadowaki H, Tobe K, Mori Y, Sakura H, Sakuta R, Nonaka I, Hagura R, Yazaki Y, Akanuma Y, Kadowaki T (1993) Mitochondrial gene mutation and insulin-deficient type of diabetes mellitus. Lancet 341: 893-894.

5. Katagiri $H$, Asano $T$, Ishihara $H$, Inukai $K$, Anai $M$, Yamanouchi T, Tsukuda K, Kikuchi M, Kitaoka H, Ohsawa N, Yazaki Y, Oka Y (1994) Mitochondrial diabetes mellitus: prevalence and clinical characterization of diabetes due to mitochondrial tRNA Leu (UUR) gene mutation in Japanese patients. Diabetologia 37: 504-510.

6. Goto Y, Nonaka I, Horai S (1990) A mutation in the tRNALeu (UUR) gene associated with the MELAS subgroup of mitochondrial encephalomyopathies. Nature 348: 651-653.

7. Sakuta R, Goto Y, Horai S, Nonaka I (1993) Mitochondrial DNA mutations at nucleotide positions 3243 and 3271 in mitochondrial myopathy, encephalopathy, lactic acidosis, and stroke-like episodes: a comparative study. J Neurological Sciences 115: $158-160$.

8. Garland H (1955) Diabetic amyotrophy. British Medical Journal 26: 1287-1290.

9. Hamilton CR, Dobson HL, Marshall J (1968) Diabetic amyotrophy: clinical and electronmicroscopic studies in six patients. Am J Med Sci 256: 81-90.

10. Asbury AK (1977) Proximal diabetic neuropathy. Ann Neurol 2: 179-180.

11. Williams IR, Mayer RF (1976) Subacute proximal diabetic neuropathy. Neurology 26: 108-116.

12. Gregersen G (1969) Diabetic amyotrophy. A welldefined syndrome? Acta Med Scand 185: 303-310.

13. Nielsen ER, Harmsen A, Vorre P (1977) Ultrastructure of muscle biopsies in recent, short-term and long-term juvenile diabetes. Acta Neurol Scandinav 55: 345-362.

14. Bresolin N, Doriguzzi C, Ponzetto C, Angelini C, Moroni I, Castelli E, Cossuta E, Binda A, Gallanti A, Gabellini S, Piccolo G, Martinuzzi A, Ciafaloni E, Arnaudo E, Liciardello L, Carenzi A, Scarlato G (1990) Ubidecarenone in the treatment of mitochondrial myopathies: a multi-center double-blind trial. J Neurol Sci 100: 70-78. 